\title{
Chinese Job Well-Being: Its Concept and Scale Developing ${ }^{1}$
}

\author{
Zhang Xinggui \\ Guangdong University of Foreign Studies \\ Guo Yang ${ }^{2}$ \\ National University of Singapore \\ David Ming Liu \\ George Fox University \\ Zhang Zhanpeng \\ Fu Jen Catholic University
}

The aim of this paper is to construct a scale of job well-being based on Chinese culture. The following steps were involved for this construction. First, we reviewed the literature on context free well-being and job well-being from western perspectives (e.g., hedonism, eudemonism) as well as Chinese culture. Then we completed a qualitative study (interviews, focus group discussions) and proposed a preliminary job well-being model. A preliminary scale was developed based on the job well-being model and existing scales in related fields. Further, we conducted another quantitative study to verify and modify the qualitative findings. We finally established a 7-dimension scale for Chinese job well-being. It has satisfactory reliability and validity, suggesting its potential applicable to Chinese culture. Despite being similar to the western model in some dimensions (e.g., intrinsic satisfaction, job competence), this job well-being model has some dimensions reflecting the uniqueness of Chinese culture (e.g., harmony, recognition from others). Moreover, high positive affect and autonomy, which are important for well-being in the west, are not obvious in this model. The different findings from China suggest the significance of conducting indigenous job well-being studies.

\section{INTRODUCTION}

Although China has enjoyed rapid economic growth for decades, findings of International Well-being Study $(1990,1995,2001)$ showed that Chinese well-being has decreased during the past years. According to the national survey by China HRD (China Human Resources Development Network 2008), 54\% of respondents experience job burnout, $72 \%$ are cynical, $27 \%$ suffer from serious emotional exhaustion, and $42.3 \%$ of the people lack sense of achievement. Lack of job well-being has impaired people's work and 
life quality tremendously and thereby induced various deviant work behaviors, which has garnered considerable attention globally. Since people on average spend a quarter of time on jobs (Warr, 2005), it is critical to investigate how individuals can experience happiness and well-being from the jobs.

Despite being an old topic in philosophy, sociology, and psychology, well-being has not gained much attention in the organizational area until the rise of positive organizational behavior. Accompanied with the positive organizational behavior movement, job well-being has witnessed a rapid progress. Scholars pay much attention to the concept of happiness, its measurement, antecedents and results. However, limitations still exist in this area. There is no agreement on defining and measuring job well-being, thus preventing the accumulation of comparative data and deterring the development in this area (Grawitch, Gottschalk \& Munz, 2006). One stream of research adopts the hedonic view and suggests that well-being consists of pleasure and happiness (Kahneman, 1999), whereas the other stream takes the eudemonic view by assuming that well-being is fulfilling oneself, exploiting one's potential, and fully functioning (Ryan \& Deci, 2001). We argue that the two streams are complementary rather than contradictory, because each offers a unique viewpoint and to integrate both streams helps us understand the whole picture of well-being. However, there are only few studies on job well-being combining both hedonic and eudemonic perspectives (Horn, 2004).

Additionally, the majority of prior job well-being studies were conducted in the western and individualist societies such as Europe and North American. Since most well-being theories and measures are based on the individualistic culture, they may not be applicable to Chinese culture, which emphasizes collectivism and harmony. As such, it is important to take the indigenous study because the existing theories are not necessarily universal and may often represent the cultural traditions of Europe and North America (Kim \& Berry, 1993).

Given the gaps mentioned above, the current study aims at exploring the structure of job well-being based on the Chinese context. We acknowledge the findings based on western and individualistic societies by integrating both hedonic and eudemonic perspectives of well-being. On the other hand, we emphasize cultural influence by drawing on studies in the Chinese context. This research will contribute to theoretical progress by providing a measure for Chinese job well-being. Moreover, it is a practical tool for managers to measure and increase job well-being of employees. Since Chinese government aims at constructing a harmonious society and enhancing well-being of the people, our study offers the index for creating the high well-being workplaces.

\section{THEORETICAL BACKGROUND}

\section{The Definition and Measure of Context-free Well-being and Job Well-being}

Well-being consists of context-free and domain-specific well-being (Warr, 2007). Job well-being, the application of well-being to a work setting, is domain-specific well-being. Literature on context-free well-being can act as the theoretical guide to study the domain-specific well-being. Just as context-free well-being can be studied from the hedonic or (and) the eudemonic perspective, job well-being scales can be developed based on hedonic or eudemonic view, or both.

Hedonic scales. Proponents of hedonic view use the term "Subjective Well-being (SWB)" by Diener (1984), which is defined as cognitive and affective reactions toward life (Diener, Suh, Lucas \& Smith, 1999). Context-free well-being scales that tap cognitive or affective evaluations include Satisfaction with Life Scale and Positive and Negative Affect Schedule (PANAS) (Diener, Emmons, Larsen \& Griffin, 1985; Watson, Clark \& Tellegen, 1988). Job-related scales based on hedonic view or (and) SWB cover the measures such as job satisfaction and job burnout (Warr, 2007). Those scales typically consider negative affect as the sign of low well-being. However, simply equating negative affect to low well-being is misleading because mentally healthy individuals may also often experience negative affect (e.g., anxiety) due to pursuit of challenging goals. Moreover, people can experience high positive affect (e.g., enthusiasm) and high negative affect (e.g., anxiety) simultaneously (Warr, 2005). 
Eudemonic scales. Eudemonic advocators suggest that happiness can be more than evaluations in terms of pleasure. For example, Ryff and Keyes (1995) assumed that well-being contains aspects of positive functioning and requires efforts and disciplines that may be at odds with short-term happiness. That is to say, with the positive functioning goal, individuals can have high well-being accompanied with negative effect. They proposed the construct psychological well-being measured by 6 dimensions (autonomy, environmental mastery, personal growth, positive relations with others, purpose in life, self-acceptance). Regarding job-domain scales, the measures that tap intrinsic job satisfaction are based on eudemonic view. Some context-free constructs (e.g., competence, aspiration, autonomy) can also be measured with a job-specific focus (Warr, 2007). Despite wide recognition of eudemonism in context-free setting, eudemonic-based job-related scales do not fulfil the purpose satisfactorily.

Scales combining both views. Well-being can be considered as a multi dimensional phenomenon encompassing both hedonic and eudemonic views (Ryan \& Deci, 2001). Warr (2007) proposed a three-dimensional approach to well-being, covering both hedonic (through pleasure and arousal) and eudemonic (through self-validation) elements. Horn (2004) developed a job well-being scale with 5 subscales (affective well-being, motivation, social well-being, cognitive well-being, psychosomatic well-being). However, there are still very few job-related scales integrating both hedonic and eudemonic views.

\section{Determinants of Job Well-being}

Job well-being is typically considered as determined by either environmental (e.g., job features, social support) or personal (e.g., personality, ways of thinking) features. As for the environment-centered perspective, job well-being scales should measure employees' evaluations on environmental factors (e.g., job features, leader behaviors). In contrast, the person-centered perspective suggests that job well-being scales should focus on personal features (e.g., self-efficacy). In recent years, however, scholars have realized that only one single angle cannot delineate the whole picture. Instead, they have increasingly emphasized the interplay between environmental and personal factors.

Environmental features. Job features have long been the focus in job well-being research. Models such as Job Characteristics Model (Hackman \& Oldham, 1976), Demand-Control- Support Model (Karasek \& Theorell, 1990), and Demand-Resource Model (Bakker \& Demerouti, 2003) are widely recognized.

Other factors such as social support, leader behaviors, and work-family conflict have also significant influences on employee well-being (Cohen \& Wills, 1985; Green-haus, Allen \& Spector, 2006; Tepper, 2007). Warr $(2005,2007)$ identified 12 environmental antecedents of job well-being: opportunity for personal control, opportunity for skill use, externally-generated goals, variety, environmental clarity, contact with others, availability of money, physical security, valued social position, supportive supervision, career outlook, and equity. While most studies examine only linear relationships between the environment and well-being, some results indicate that non-linear relationship may occur for certain aspects of the environment. For instance, the "vitamin model" (Warr, 2007) suggests that an absence of the primary environmental characteristics may reduce well-being, but their presence beyond a certain level may not further increase well-being.

Personal features. The person-centered view assumes that individual differences such as demographic characteristics, personality, or ways of thinking account for well-being being independent of environmental features. The meta-analytic results indicated that personality factors account for $39 \%$ $63 \%$ of SWB variances (Steel, Schmidt \& Shultz, 2008). In terms of job settings, the meta-analysis showed that the multiple correlation between big five personality and job satisfaction is .41 (Judge, Heller \& Mount, 2002). Connolly and Viswesvaran (2000) found that mean correlations of job satisfaction with positive affectivity and negative affectivity are .49 and -.33 respectively. Other personality traits (e.g., optimism, hardiness) have also garnered attention (e.g., Kobasa, Maddi \&Kahn, 1982; Scheier, Carver \& Bridges, 2001). 
Joint effects of environmental and personal features. While some researchers assume that either environmental or personal features predispose individuals to have a certain well-being level regardless of other factors, others believe that environmental and personal factors jointly affect job well-being, either additively or interactively (Judge, Bono \& Locke, 2000; Warr, 1999). For instance, the positive correlation between job variety and job satisfaction is stronger for employees with high need for growth than for those with low need for growth (Warr, 2005). The person-organization fit, which can be understood as a specific type of person-situation interaction involving the match between corresponding person and environment dimensions, has maintained a prominent position in organizational psychology and related fields (Edwards, 2008).

\section{Well-being in the Chinese Context}

Since well-being is rooted in the cultural background, the impacts of Chinese culture need to be emphasized when studying Chinese job well-being. Rather than the western individualistic culture, which pursues environmental mastery, hedonism, and self-actualization, the Chinese collectivist culture emphasizes adapting to environments, playing individual's social role, and harmony between people and environments (Wong \& Zheng, 2008). As such, we need to understand how the well-being perspectives of Chinese people are shaped by the Chinese culture.

Perspectives of Chinese culture. Confucianism, Taoism and Chinese Buddhism, the roots of traditional culture and beliefs of Chinese people, all advocate that well-being is some kind of a harmonious state. Confucian philosophy views interpersonal harmony as well-being and tells individuals to keep smooth with others when persisting their own ways. Taoism considers the harmony between self and environments (nature, universe). For Chinese Buddhism, real well-being lies in a peaceful and simpatico heart (Wong \& Zheng, 2008).

In alignment with the harmony-oriented tradition, Chinese culture also regards well-being as a carefree state with no negative effect, rather with high positive effect. While western scholars underemphasized the dark side of positive effect until recently (Gruber, Mauss \& Tamir, 2011), traditional Chinese avoid extreme emotions and believe that extreme joy begets sadness. Instead, they advocate restraining from extreme emotions, both negative and positive (Zeng \& Guo, 2012). Kuppens, Realo and Diener (2008) found that in some East Asian cultural contexts, the hedonic aspects predict well-being outcomes to a much weaker degree or not at all.

\section{Well-being Studies for Chinese.}

$\mathrm{Lu} \&$ Shih (1997) found that Taiwan Chinese have six unique sources of well-being as compared with westerners: harmonious relations with relatives and friends, recognition from others, better life than others, accepting fate and being happy about it, material satisfaction, and work achievement. Based on mainland Chinese, Xing(2005) found a 10-dimensional model of well-being: economic contentment, mental health, confident for society, sense of growth, sense of meaningfulness, self-acceptance, physical well-being, balanced state of mind, positive relations with others, and harmonious family. The results reflect the collectivist culture because some well-being dimensions are closely related with other people or the society and the need for autonomy has not been found as a component. Moreover, instead of viewing well-being as highly positive effect, Chinese value the balanced state of mind or being happy about fate.

Job well-being studies for Chinese. Majority of job well-being studies in China focus on job satisfaction. For instance, the dimensions of job satisfaction (e.g., leader behaviors, rewarding, and collaboration) were examined. Scholars have begun to attend to job well-being in recent years. Wang and $\mathrm{Hu}$ (2006) summarized the western literature on job well-being. Li \& Li $(2007,2010)$ discussed the values of happiness management. There are also empirical well-being studies based on a working context (e.g., Feng, Lu \& Xiao, 2008). However, measures used in these empirical studies, which are mostly developed based on westerners, may not be adaptable to Chinese context. 
Conjectures about contours of job well-being for Chinese: Since the theories and measures of job well-being are still not compatible to Chinese context. To guide the following qualitative as well as quantitative studies, we have some conjectures based on prior research and Chinese culture.

While the hedonism in western literature emphasizes high positive effect, Chinese do not view high positive effect as necessary. Instead, they advocate for maintaining the psychological balance (e.g., moderate positive effect, low negative effect). As for eudemonism, intrinsic job factors and positive social relationships are also highly valued by Chinese. While traditional Chinese do not regard job autonomy as important, the employees of the new generation, especially those in the metropolis, may value autonomy. The collectivist culture suggests that Chinese need not just sense of competence but also recognition from others. Chinese also need harmonious relationships with people and things around (e.g., colleagues, family, job, company, society, nature). Since Chinese pursue harmony in various aspects, they typically maintain work-life balance rather than overworking. In addition, monetary incentives are still critical in developing countries like China. However, the collectivist culture suggests that Chinese may be more affected by the comparison with others than by the absolute amount of income. As happiness is a dimension of personality, in Chinese notion, hardiness and optimism (perseverance, optimism and tolerance) are considered to be very important to people's life. Thus, we propose that the dimensions of Chinese job well-being include harmony, recognition from others, work-life balance, intrinsic satisfaction, pay satisfaction, job competence, and hardiness and optimism.

\section{CURRENT STUDY}

\section{Procedure and Method}

Given the importance of research on job well-being and lack of measures applicable to Chinese context, the current study focuses on exploring the dimensions of Chinese job well-being. The study refers to indigenous psychology, which emphasizes situated knowledge and argues that dominant conceptions can systematically misrepresent other cultures or subordinated groups. Based on this perspective, it is necessary to examine the knowledge and beliefs people have in their natural contexts (Hwang, 2013). In order to focus on the perspectives of local people and allow local concepts and values to emerge, a qualitative approach is useful. A quantitative study is also employed to test, adapt, and generalize the qualitative findings.

\section{Qualitative Study}

We conducted open-ended interviews and focus group discussions (8 to 10 employees for each group) to collect perspectives (e.g., components and sources of job well-being) from employees in Guangzhou or Shenzhen, mainland China. During this study, 23 one-to-one interviews and two focus group discussions were conducted from full-time employees of different ages, genders, occupations, job levels, and enterprise ownerships. By integrating literature reviews, interviews, and focus group discussions, we proposed a 12-dimensional model of job well-being as a pilot study for the scale development in the quantitative study (see the Results).

\section{Quantitative Study-Pretest Survey and Data Analysis}

Participants. For this study, 148 full-time employees (68women, 64 married, age range: 21-55 years, 130 with college degrees or above) were involved. The respondents worked in companies, governments, or schools of Shenzhen or Guangzhou.

Sources of job well-being scale for pre-test survey. Before the pre-test, we developed the preliminary job well-being scale. The scale consisted of 125 items, which were from three sources: written by authors according to the concept of each structure; interview and focus group discussion records; well-developed scales in similar fields (e.g., job satisfaction, context-free well-being, organization commitment). The pre-test also included the single-item job well-being scale "When I consider my job in general, I think I'm 
an employee with high well-being". All items were rated with the Likert-type scale from 1 ("strongly disagree") to 5 ("strongly agree").

To make items clearly understood, we requested several employees with high school education level to point out inadequate words or sentences. In addition, a professor in OBHR and a human resources manager separately evaluated each item's content. Items regarded as unsuitable by both raters would be deleted.

Data Analysis. Items were excluded by stepwise method and by the following item-analysis criteria: (a) The correlation coefficient between the item and the total score was significant; (b) Items with standard deviation $(S D)$ near 1.0; (c) Items with insignificant $C R$ value. Based on the criterion above, we kept 65 items, which would be used in the formal survey.

\section{Quantitative Study-Formal Survey and Data Analysis.}

Participants. The participants of the formal survey included 442 full-time employees ( 242 women, 228 married, age range: 21-55 years, 392 with college degree or above). We conducted the exploratory factor analysis (EFA) for one half of the current sample and confirmatory factor analysis (CFA) for the other half.

Job well-being scale for formal survey. The scale consisted of the remaining 65 items (including the single-item job well-being scale) after item analysis of pre-test survey. We also modified the wording of some items to make them more clearly understandable.

Data analysis. When running EFA, we deleted the items based on the following criteria: (a) items with factor loadings is above 0.40 on each factor; (b) The correlation coefficient between the title and the total score was significant; (c) items belong to the factor that has more than three items; (d) items with $S D$ near 1.0; and (e) items with insignificant $C R$ value. Finally, 34 items (seven factors, cumulative \% $=67.9 \%$ ) were kept to form the final scale (see Appendix).

\section{RESULTS}

\section{Preliminary Model of Chinese Job Well-being Based on Qualitative Study}

We extracted 7 factors from the remaining 34 items, and the 7 factors accounted for $68 \%$ of variance.

TABLE 1

EXPLORATORY FACTOR ANALYSIS FOR JOB WELL-BEING MODEL (N=221)

\begin{tabular}{|c|c|c|c|c|c|c|c|}
\hline Items & Factor1 & Factor2 & Factor3 & Factor4 & Factor5 & Factor6 & Factor7 \\
\hline 1 & 0.864 & & & & & & \\
\hline 2 & 0.850 & & & & & & \\
\hline 3 & 0.840 & & & & & & \\
\hline 4 & 0.812 & & & & & & \\
\hline 5 & 0.795 & & & & & & \\
\hline 6 & & 0.801 & & & & & \\
\hline 7 & & 0.795 & & & & & \\
\hline 8 & & 0.718 & & & & & \\
\hline
\end{tabular}




\begin{tabular}{|c|c|c|c|c|c|c|c|}
\hline Items & Factor1 & Factor2 & Factor3 & Factor4 & Factor5 & Factor6 & Factor7 \\
\hline 9 & & 0.703 & & & & & \\
\hline 10 & & 0.647 & & & & & \\
\hline 11 & & 0.641 & & & & & \\
\hline 12 & & 0.584 & & & & & \\
\hline 13 & & 0.484 & & & & & \\
\hline 14 & & & 0.873 & & & & \\
\hline 15 & & & 0.870 & & & & \\
\hline 16 & & & 0.845 & & & & \\
\hline 17 & & & 0.804 & & & & \\
\hline 18 & & & 0.803 & & & & \\
\hline 19 & & & & 0.728 & & & \\
\hline 20 & & & & 0.670 & & & \\
\hline 21 & & & & 0.655 & & & \\
\hline 22 & & & & 0.654 & & & \\
\hline 23 & & & & 0.536 & & & \\
\hline 24 & & & & 0.406 & & & \\
\hline 25 & & & & & 0.764 & & \\
\hline 26 & & & & & 0.742 & & \\
\hline 27 & & & & & 0.624 & & \\
\hline 28 & & & & & & 0.738 & \\
\hline 29 & & & & & & 0.727 & \\
\hline 30 & & & & & & 0.571 & \\
\hline 31 & & & & & & 0.550 & \\
\hline 32 & & & & & & & 0.759 \\
\hline 33 & & & & & & & 0.684 \\
\hline 34 & & & & & & & 0.444 \\
\hline
\end{tabular}

Note: We conducted Varimax rotation. All loadings above 0.40 are shown. 
We named each factor according to the content of items belonging to each factor. The seven dimensions are as following:

Pay satisfaction. It includes 5 items, which concern to the satisfaction with such as salary, welfare, and rewarding system.

Hardiness and optimism. 8 items were retained in this factor. Items involve perseverance, independence, optimism, tolerance and so on.

Work life balance. This dimension assesses the effects of the job on emotions, social life and leisure. For this, 5 items were extracted. Notably, the item of work-family is not included in this dimension but in the dimension engagement and competence. The possible explanation is that employees may feel incompetent when they cannot balance work and family.

Intrinsic satisfaction. This dimension has 6 items, which ask respondents about the intrinsic motivators such as meaningfulness of job, sense of achievement, growth, and self-actualization.

Harmony. 3 items are included, which concern to open climate, satisfaction with freedom of job, and leader-member relationships.

Recognition from others. With 4 items, this dimension asks whether employees gain recognition from organizations or leaders, and whether employees are proud of their jobs or not when in front of others.

Job Competence. This dimension includes 3 items, which ask employees' feeling of competence about their jobs.

\section{Confirmatory Factor Analysis}

Confirmatory factory analysis (CFA) results indicated that our proposed 7-dimensional model fit the data well, with CFI, RMR, and RMSEA of $.96, .076$, and .064 respectively. We also compared our 7-dimensional model with other alternatives such as 5-dimensional model or 6- dimensional model. As shown in Table 2, the chi-squares of alternative models were significantly greater than that of the baseline 7-dimensional model. Moreover, the 7-dimensional model demonstrated higher CFI and lower RMR and RMSEA than the alternatives, suggesting that our proposed baseline model fit data better than other alternatives.

TABLE 2

CONFIRMATORY FACTOR ANALYSIS FOR JOB WELL-BEING MODEL (N=221)

\begin{tabular}{|l|r|c|c|c|c|c|}
\hline \multicolumn{1}{|c|}{ Models } & \multicolumn{1}{c|}{ Model $\boldsymbol{\chi}^{\mathbf{2}}$} & d.f. & $\boldsymbol{\chi}^{\mathbf{2}} / \mathbf{d . f .}$ & CFI & RMR & RMSEA \\
\hline Baseline 7-factor model & 962.83 & 506 & 1.90 & 0.96 & 0.076 & 0.064 \\
\hline 5-factor model & 1082.39 & 475 & 2.28 & 0.87 & 0.093 & 0.090 \\
\hline 6-factor model & 1032.53 & 480 & 2.15 & 0.95 & 0.088 & 0.072 \\
\hline
\end{tabular}

\section{Reliability and criterion validity}

Our final scale had good reliability and criterion-validity. The Cronbach $\alpha$ of the overall job well-being scale (excluding the single-item job well-being scale) and the seven subscales were $.928, .932, .875, .904, .882, .852, .713$, and .708 respectively (Table 3). Moreover, the correlation between the overall job well-being scale (excluding the single-item job well-being scale) and the single-item job well-being scale was .694, suggesting satisfactory criterion validity (Table 4). 
TABLE 3

\section{RELIABILITY OF JOB WELL-BEING MEASURES}

\begin{tabular}{|l|c|c|}
\hline Measures & Number of items & Cronbach $\boldsymbol{\alpha}$ \\
\hline Overall job well-being scale & 34 & .928 \\
\hline Pay satisfaction subscale & 5 & .932 \\
\hline Hardiness and optimism scale & 8 & .875 \\
\hline Work and life balance scale & 5 & .904 \\
\hline Intrinsic satisfaction scale & 6 & .882 \\
\hline Harmony scale & 3 & .852 \\
\hline Recognition from others scale & 4 & .713 \\
\hline Job competence scale & 3 & .708 \\
\hline
\end{tabular}

TABLE 4

CRITERION-VALIDITY OF JOB WELL-BEING SCALE WITH OTHER SCALES

\begin{tabular}{|l|c|}
\hline \multicolumn{1}{|c|}{ Measures } & Correlation with job well-being scale \\
\hline Single-item job well-being & .694 \\
\hline Single-item job satisfaction & .660 \\
\hline
\end{tabular}

\section{DISCUSSION}

\section{Theoretical Implications}

Seven dimensions of job well-being are proposed according to EFA, namely pay satisfaction, hardiness and optimism, work-life balance, intrinsic satisfaction, democracy and harmony, recognition from others, and job competence. Although the current sample has high salaries and good educational background as compared to the average employee in the selected cities, pay satisfaction is also the critical component of job well-being. Future research can examine whether such result is due to the relative inadequate welfare system in China.

The factor of hardiness and optimism, albeit being ignored in my job well-being studied, is found to be an important dimension in this study. Such finding suggests that job well-being not only depends on objective environments, but also on personal features (e.g., personality, belief). Those high in hardiness and optimism will still strive for excellence even confronted with difficulty and frustration. More research is needed to examine the antecedents and outcomes of this factor.

We should note that the indicators of job well-being are developed according to the pattern (e.g., cognitive, emotional, hedonic, eudemonic, dispositional component). Instead, each indicator can be a mixture of the above components since a person's certain feelings or judgments often consist of varied factors. For example, harmony is still regarded as an important component, but harmony cannot be maintained without an open climate. As such, the openness is classified into the dimension harmony.

As suggested by positive psychologists, previous studies mostly focus on negative side (e.g., stress, burnout). As a result, scholars understand a lot about how people endure under adversity (e.g., Benjamin, 1992; Smith, 1997) but know very little about how normal people flourish under more benign conditions (Seligman, Csikszentmihalyi, 2000). As such, this study will promote organizational research and practice from the view of positive psychology. 


\section{Practical Implications}

Through measuring job well-being with this 7-dimension scale, practitioners can find the sources of employees' low well-being and make improvements according to characteristics of the employees as well as the company rather than imitating other firms blindly. Despite academic study on job well-being has lagged behind; it is a hot topic in the practical field. A nationwide survey in 2008 by China HRD showed that nearly 30 percent of 5350 Chinese employees had rather low job well-being and only less than 10 percent scored high, suggesting the necessity of assessing and improving employee well-being level (China Human Resources Development Network, 2008). Since the well-being index of the people serves as the foundation of the harmonious society that is pursued by the Chinese government, it is the mutual benefits of the employees, enterprises, as well as the government to enhance the well-being of the people.

\section{Limitations and Future Directions}

This research work is exploratory and the number of items on each factor is not identical. Some factors have 8 items, while others have only 3 items. In the future, we should verify and revise the scale continuously. The relation between each job well-being dimension and other variables (e.g., personality, job features) also deserves attention. In addition, we only surveyed employees in the most developed cities of southeast China. We should test the applicability of the job well-being structure in other places of China.

\section{CONCLUSION}

The final scale of Chinese job well-being has 7 dimensions and demonstrates satisfactory reliability and validity, suggesting its potential for measuring the job well-being of Chinese employees.

Over the years Job Well-being research has been expanded to the areas including Physical Well-being, Health Well-being, and Social Well-being / Relationships Well-being and caught attention of numerous Scholars (Peccei, 2004; Van De Voorde et al. 2012; Kooij et al. 2013). Physical Well-being relates to employees' personal experience in a subjective manner regarding their psychological and physical conditions. It involves employees' psychological and physical health as well. What's more, Social Well-being is intertwined into employees Social Relationships with employers or coworkers as well as trust, social support, mutual benefits, leader member exchange, and cooperation (Grant et al. 2007; Van De Voorde, 2009; Kooji et al. 2013). The constant focus and research on Social Well-being by western scholars depicts the importance of Social Relationship in job market and echoes the findings in said research in the dimensions of harmony and recognition from others.

Despite being similar as the western model in some dimensions (e.g., intrinsic satisfaction, job competence), this job well-being model has some dimensions reflecting the uniqueness of Chinese culture (e.g., harmony, recognition from others). Moreover, high positive effect and autonomy, which are important for well-being in the west, are not obvious in this model. The different findings between China and west also suggest the significance of conducting indigenous job well-being studies.

This research concludes that the 7 Dimensions in the Chinese Employees Job Well-being can be studied in the same level and even divided into more detailed levels. Whether or not there are interactional relationships related to each dimension needs further research and theoretical development.

\section{ENDNOTES}

1. This study was supported by a grant from China National Social Science Fund (14BGL076) and a major breeding project from Guangdong University of Foreign Studies (15Z3).

2. Corresponding author, email: y.guo@u.nus.edu 


\section{REFERENCES}

Bakker, A. B., Demerouti, E., Boer, E. D., \& Schaufeli, W. B. 2003. “Job Demands and Job Resources as Predictors of Absence Duration and Frequency." Journal of Vocational Behavior, 62(2): 341-356.

Benjamin, L. T. (Ed.). 1992. "The History of American Psychology [Special issue].” American Psychologist, 47(2).

Connolly, J. J. \& Viswesvaran, C. 2000. "The Role of Affectivity in Job Satisfaction: A Meta-analysis." Personality and Individual Differences, 29: 265-281.

China Human Resources Development Network. 2008. "2008 China Workplace Burnout Survey Report." Accessed August 20, 2018. http://finance.sina.com.cn/leadership/zyfz/20080519/18084885234.shtml

Diener, E. 1984. "Subjective Well-being." Psychological Bulletin 95: 542-575.

Diener, E., Emmons, R. A., Larsen, R. J., \& Griffin, S. 1985. "The Satisfaction With Life Scale.” Journal of Personality Assessment, 49(1): 71-5.

Diener, E., Suh, E., Lucas, R. E. \& Smith, H. 1999. "Subjective Well-being: Three Decades of Progress." Psychological Bulletin 125: 273-302.

Edwards, J. R. 2008. "Person-environment Fit in Organizations: An assessment of Theoretical Progress." The Academy of Management Annals 2:167-230.

Feng, D. D., Lu, C. Q., \& Siu, A. L. 2008. "The Relationships Among Job Insecurity, Well-being and Performance: Role of Self-efficacy.” Acta Psychologica Sinica 40(4): 448-455.

Grawitch, M. J., Gottschalk, M. \& Munz, D. C. 2006. "The Path to a Healthy Workplace.” Consulting Psychology Journal: Practice and Research 58(3): 129-147.

Green F. 2006. Demanding Work: The Paradox of Job Quality in the Affluent Economy. Princeton University Press.

Gruber, J., Mauss, I. B., \& Tamir, M. 2011. "A Dark Side of Happiness? How, When, and Why Happiness is Not Always Good." Perspective on Psychological Science 6: 222-233.

Hackman, J. R. \& Oldham, G. R. 1976. "Motivation Through the Design of Work: Test of a Theory." Organizational Behavior and Human Performance 16: 250-279.

Horn, J. E. V., Taris, D. T. W., Schaufeli, W. B., et al. 2004. "The Structure of Occupational Well-being:A Study Among Dutch Teachers.”Journal of Occupational \& Organizational Psychology 77(3): $365-375$.

Hwang, K. K. 2013. "The Construction of Culture-inclusive Theories by Multiple Philosophical Paradigms." Social Epistemology Review and Reply Collective 2: 46-58.

Judge, T. A., Bono, J. E. \& Locke, E. A. 2000. "Personality and Job Satisfaction: The Mediating Role of Job Characteristics."Journal of Applied Psychology, 85:237-249.

Judge, T. A., Heller, D. \& Mount, M. K. 2002. "Five-facto Model of Personality and Job Satisfaction: A Meta- Analysis." Journal of Applied Psychology 87(3): 530-541.

Kahneman, D., Diener, E. \& Schwarz, N. 1999. Well-being: The Foundation of Hedonic Psychology. New York: Russell Sage Found.

Karasek, R. A. \& Theorell, T. 1990. Healthy Work: Stress, Productivity and Reconstruction of Working Life. New York: Basic Books.

Kim, U. \& Berry, J. 1993. Indigenous Psychologies: Research and Experience in Cultural Context. Newbury Park, CA: Sage.

Kobasa, S. C., Maddi, S. R. \& Kahn, S. 1982. "Hardiness and Health: A Prospective Study.” Journal of Personality and Social Psychology 42: 168-177.

Kooij D T A M, Guest D E, Clinton M, Knight T, Jansen P G and Dikkers J S. 2013. "How the Impact of HR Practices on Employee Well-being and Performance Changes With Age." Human Resource Management Journal, 23(1): 18-35. 
Kuppens, P., Realo, A., \& Diener, E. 2008. "The Role of Positive and Negative Emotions in Life Satisfaction judgment across nations." Journal of Personality and Social Psychology 95: 66-75.

Lu, L. \& Shih, J. B. 1997. "Sources of Happiness: A Qualitative Approach.” Journal of Social Psychology, 137(2): 181-187.

Peccei R E. 2004. Human Resource Management and the Search for the Happy Workplace. Erasmus University Rotterdam.

Ryan, R. M. \& Deci, E. L. 2001. "On Happiness and Human Potentials: A review of Research on Hedonic and Eudemonic Well-being." Annual Review Psychology 52:141-166.

Ryff, C. D. \& Keyes, C. L. M. 1995. "The Structure of Psychological Well-being Revisited.” Journal of Personality and Social Psychology 69:719-727.

Scheier, M. F., Carver, C. S., \& Bridges, M. W. 2001. "Optimism, Pessimism, and Psychological Well-being." In Optimism \& pessimism: Implications for theory, research, and practice, edited by E. C. Chang, 189-216. Washington, DC: American Psychological Association.

Seligman, M. E. P., \& Csikszentmihalyi, M. 2000. "Positive Psychology: An Introduction." American Psychologist 55 (1): 5-14.

Smith, R. 1997. The human sciences. New York: Norton.

Van de Voorde K. 2009. "HRM, Employee Well-being and Organizational Performance: A Balanced Perspective." Ridderprint Offsetdrukkerij BV.

Van De Voorde K, Paauwe J and Van Veldhoven M. 2012. "Employee Well - being and the HRM Organizational Performance Relationship: A Review of Quantitative Studies." International Journal of Management Reviews 14(4): 391-407.

Wang, J. Y., \& Hu, A. A. 2006. "Review of Research on Subjective Job Well-being." Foreign Economics and Management 28 (8): 49-55.

Warr, P. B. 1990. "The Measurement of Well-being and Other Aspects of Mental Health." Journal of Occupational Psychology 63: 193-210.

Warr, P. B. 1999. "Well-being and the Workplace." In Well-being: The foundations of Hedonic Psychology, edited by D. Kahneman, E. Diener \& N. Schwarz. New York: Russell Sage Foundation.

Warr, P. B. 2005. Handbook of Work Stress. Thousand Oaks. CA: Sage.

Warr, P. B. 2007. Work, Happiness, and Unhappiness. London: Lawrence Erlbaum Associations.

Watson, D., Clark, L. A., \& Tellegen, A. 1988. "Development and Validation of Brief Measures of Positive and Negative Affect: The PANAS Scales." Journal of Personality and Social Psychology 54(6): 1063-1070.

Wong, F.Y., \& Zheng, H. 2008. Chinese Cultural Psychology. Guangzhou: Jinan University Press.

Xing, Z. J. 2005. Measuring Well-being. Beijing: Renmin Press. 


\section{APPENDIX \\ CHINESE JOB WELL-BEING SCALE}

\section{Items}

\section{Pay Satisfaction}

1 The pay system is satisfactory.

2 The pay system can motivate people to work hard.

3 I am very satisfied with my current income.

4 I am satisfied with my income when concerning my skills and efforts in the job.

5 I am satisfied with the opportunity to have a rise in income.

\section{Hardiness and Optimism}

6 Life is filled with challenge, but there are always ways to work out most problems.

7 I make the greatest effort to do my job despite I may be confronted with difficulty or failure.

8 I will do whatever I regard as valuable no matter how others may think about.

9 I firmly believe that efforts will lead to good results.

10 It is very important for me to have a high level of autonomy in the job.

11 I possess (or will possess) the knowledge, capability, and experience required by the job.

12 The current pay can cover my daily expenses.

13 I can always be tolerant toward others during the job.

\section{Work Life Balance}

14 I am always in bad mood because of the job.

15 I always feel tired on the job.

16 I can hardly enjoy life because of the job.

17 I can hardly have time or energy to get along with friends because of the job.

18 I always feel bored on the job.

\section{Intrinsic Satisfaction}

19 I can attain sense of achievement from the job.

20 I have the opportunity to make use of my strengths during the job.

21 I can make progress and accumulate knowledge and experience from the job.

$22 \mathrm{My}$ job is very meaningful to me.

23 I am very satisfied with the stability with the job.

24 I am making progress toward the target on the job.

\section{Harmony}

25 I am very satisfied with the autonomy of the job.

26 I like the open atmosphere in the organization very much.

27 The leaders have positive relationship with employees in the organization.

\section{Recognition From Others}

28 The leaders appreciate my job.

29 The organization appreciates the work I have done.

30 The organization ignores most of my efforts on the job.

31 I am very proud when talking about my job with my friends or others. 


\section{Job Competence}

32 Usually I can attain the target on the job energetically.

33 The job is pleasant on the whole despite sometimes I may be worried or nervous.

34 I cannot only do my job well but also take the responsibility of my family affairs. 